\title{
CP Violation at ATLAS
}

\author{
Tatjana Jovin $^{* \dagger}$ \\ Vinča Institute of Nuclear Sciences, University of Belgrade, \\ 12-14 Mihaila Petrovića Alasa, Belgrade, Serbia \\ E-mail: Tatjana.Jovinecern.ch
}

The Standard Model predicts a tiny CP-violating effect in the $B_{s}$-meson system that can be experimentally determined by the ATLAS experiment at LHC employing a time-dependent analysis of the 'golden' $B_{s}^{O} \rightarrow J / \psi \phi$ decay channel to extract the weak mixing phase $\phi_{s}^{J / \mu \phi}$. Precise measurement of the mass and lifetime of the $B$-hadron is crucial for the measurement of the CP-violating quantities. To validate this, we test the performance of the ATLAS detector by measuring the mass and lifetime of the $B_{s}^{0}$ meson using the $B_{s}^{0} \rightarrow J / \psi \phi$ decay. In parallel, the measurement of the mass and lifetime of the $B_{d}^{0}$ meson is performed using the decay channel $B_{d}^{0} \rightarrow J / \psi K^{* 0}$ as it represents an excellent control channel.

The final state particles from $B_{s}^{0} \rightarrow J / \psi \phi$ and $B_{d}^{0} \rightarrow J / \psi K^{* 0}$ decays are reconstructed in the ATLAS detector using proton-proton collision data at a center-of-mass energy of $7 \mathrm{TeV}$ corresponding to an integrated luminosity of $40 \mathrm{pb}^{-1}$. Within the statistical uncertainties, the fitted masses and lifetimes of both $B$ mesons are consistent with the world average values.

The XIth International Conference on Heavy Quarks and Leptons,

Prague, Czech Republic

June 11-15, 2012

${ }^{*}$ Speaker.

${ }^{\dagger}$ On behalf of the ATLAS collaboration. 


\section{Introduction}

The Standard Model of particle physics (SM) predicts a violation of the CP symmetry, by including a single irreducible complex phase in the CKM matrix. All measurements of the CPviolating processes, from its discovery in neutral $K$ decays in 1964 through the recent observations in $B$ decays, proof that the CKM phase is different from zero, providing that the matrix of three-generation quark mixing is the dominant source of CP violation within the SM. However, almost all extensions of the SM imply that there are additional sources of CP violation and despite SM phenomenological success it fails to accommodate the observed matter-antimatter asymmetry in the universe by several orders of magnitude. This discrepancy suggests that nature provides additional sources of CP violation beyond the SM.

Thus, the main task of modern day physics is to test the key predictions of the SM, to search for the source of $\mathrm{CP}$ violation within it and to search for discrepancies providing evidence for physics beyond the SM that establish a connection between the observed CP violation and the one needed to explain baryon asymmetry.

At present, a promising strategy adopted by the ATLAS experiment at LHC is to study processes where the SM predicts a small $\mathrm{CP}$ violation, while $\mathrm{SM}$ extensions predict large $\mathrm{CP}$ violation effects.

\subsection{CP violation in the neutral $B_{s}^{0}$ system}

The ATLAS experiment sets its first measurement of CP violation in the $B_{s}$-meson system by studying the asymmetry of neutral meson decays into final $\mathrm{CP}$ eigenstates, using an untagged time-dependent analysis.

Flavour change via the weak interaction gives rise to $B_{s}^{0}-\overline{B_{s}^{0}}$ mixing, and consequently, the $B_{s}^{0}$ meson is found in the quantum superposition states denoted as "heavy" and "light" $\left(B_{s}^{H}\right.$ and $B_{s}^{L}$ ) which are CP eigenstates, odd and even respectively. Both states have different masses and lifetimes. The oscillation frequency of $B_{s}^{0}$ meson mixing is characterized by the mass difference $\mathrm{m}_{\mathrm{s}}$ of the $B_{s}^{H}$ and $B_{s}^{L}$ mass eigenstates and by the CP-violating phase $\phi_{s}^{\mathrm{J} / \varphi \phi}$ which arises from the interference between the decay and the mixing. Contrary to any other system, the $B_{s}^{0}$ meson is strongly mixed and thus provides a fruitful domain to search for CP-violating effects. Another physical quantity involved in $B_{s}^{0}-\overline{B_{s}^{0}}$ mixing is the decay width difference $\Gamma_{\mathrm{s}}=\Gamma_{\mathrm{L}}-\Gamma_{\mathrm{H}}$ of $B_{s}^{L}$ and $B_{s}^{H}$. Physics beyond the SM is not expected to affect $\Gamma_{\mathrm{s}}$ as significantly as $\phi_{s}^{J / \varphi \phi}$ [1]. Extracting $\Gamma_{\mathrm{s}}$ from the data is nevertheless useful as it allows theoretical predictions to be tested.

The decay $B_{s}^{0} \rightarrow J / \psi \phi$ is a pseudo-scalar to vector-vector transition. Since $J / \psi$ and $\phi$ are vector mesons they can have an even or odd relative orbital momentum $L$ in the final state $(L$ $=0,1,2)$. Due to total angular momentum conservation, the final state is therefore an admixture of CP-even $(L=0,2)$ and CP-odd $(L=1)$ states. Consequently, the decay is described by three time-dependent complex amplitudes corresponding to polarisation states of the vector mesons. The resulting admixture tends to dilute (or cancel) the overall CP asymmetry. Using an angular analysis method the different $\mathrm{CP}$ components could be separated statistically and the $\mathrm{CP}$ information can be obtained without dilution $[2,3]$. 
In the absence of $\mathrm{CP}$ violation, the $B_{s}^{H}$ state would correspond exactly to the CP-odd state and the $B_{s}^{L}$ to the CP-even state.

The main reasons why the decay $B_{s}^{0} \rightarrow J / \psi \phi$ is considered the 'golden' channel in the study of CP violation with the ATLAS experiment are:

- Theoretically clean sample. This implies that the CP-violating phase $\phi_{s}^{J / \mu \phi}$ is one of the CP observables with the smallest theoretical uncertainty;

- The phase $\phi_{s}^{J / \varphi \phi}$ is predicted to be small in the $\mathrm{SM} \sim O\left(10^{-2}\right)$ and a precise measurement of $\phi_{s}^{J / \varphi \phi}$ could therefore lead to an indirect discovery of New Physics that would contribute to the $B_{s}^{0}-\overline{B_{s}^{0}}$ box diagrams. Measurement of $\phi_{s}^{J / \varphi \phi}$ thus presents a very sensitive probe for New Physics.

In addition, the following topics make the decay channel $B_{s}^{0} \rightarrow J / \psi \phi$ interesting as well:

- Experimentally clean sample. This provides clear experimental signature and good separation from background;

- The expected sizeable width difference ( $\left.\Gamma_{\mathrm{s}}\right)$ allows the extraction of CKM phases from untagged $B_{s}^{0}$ data samples, where the rapid oscillations terms $\left(\Delta m_{s} t\right)$ cancel out [4];

- This mode has a relatively large branching fraction and with the gigantic B-hadron production rate at LHC $\left(10^{12} \mathrm{~b} \overline{\mathrm{b}}\right.$ pairs per year/experiment, during the first three years of operation) it will allow large data samples to be accumulated.

The $B_{d}^{0} \rightarrow J / \psi K^{* 0}$ channel provides a valuable testing ground for measurements of $B_{s}^{0} \rightarrow J / \psi \phi$ due to its equivalent topology and similar helicity structure of the final state, with the advantage of higher statistics. The final state of $B_{d}^{0} \rightarrow J / \psi K^{* 0}$ decay, with a subsequent decay of $K^{* 0}$ to charged mesons $K$ and $\pi$, allows the flavour of the decaying $B_{d}^{0}$ to be determined. This decay mode will therefore be used to determine the flavour tagging performance in the CP violation studies of $B_{s}^{0} \rightarrow J / \psi \phi$ decay.

\section{ATLAS detector}

\subsection{B-physics potential of the ATLAS detector}

The ATLAS experiment [5] is a general-purpose particle physics detector with a forwardbackward symmetric cylindrical geometry near $4 \pi$ coverage in the solid angle. New particle searches at the nominal LHC luminosity have defined most of the performance specification of the ATLAS detector. B-physics requirements have been accommodated in the design of the inner detector, the trigger systems and the muon system that are of particular importance of the reconstruction of $B$ meson candidates.

The inner tracking detector (ID), surrounded by a superconducting solenoid providing $2 \mathrm{~T}$ magnetic field, consists of the silicon pixel detector (Pixel), the silicon microstrip detector (SCT) and the transition radiation tracker (TRT). Inner Detector has full coverage in $\phi$ and covers the pseudorapidity range $|\eta|<2.5$, with the transverse momentum resolution of $\Delta p_{T} / p_{T}=0.04 \% \times p_{T} \oplus 2 \% \quad\left(p_{T} \quad\right.$ in $\left.\mathrm{GeV}\right)$ and the resolution of the impact parameter 
$\sigma\left(d_{0}\right)=10 \mathrm{~m}\left(p_{T}>10 \mathrm{GeV}\right)$. The primary vertex resolution for events with 70 tracks or $\sqrt{\sum_{t r k} p_{T}^{2}}$ over $8 \mathrm{GeV}$ has been measured to be $\sim 30 \mathrm{~m}$ in the transverse plane and about $50 \mathrm{~m}$ in the longitudinal direction [6]. Typical position resolutions for hits in the Pixel, SCT and TRT are 10,17 and $130 \mu \mathrm{m}$ for the $R$ - $\phi$ coordinate, respectively, and in case of the Pixel and SCT, 115 and $580 \mu \mathrm{m}$ for the $z$ coordinate.

The muon spectrometer (MS) surrounds the calorimeters and consists of three large superconducting toroids with eight coils each providing a $0.5 \mathrm{~T}$ magnetic field, a system of tracking chambers and detectors for triggering. The MS covers a pseudorapidity region of \pm 2.7 , with the transverse momentum resolution of $\sigma / p_{T}<10 \%$ for tracks up to $1 \mathrm{TeV}$, translating a sagitta along the z-axis of about $500 \mathrm{~m}$ to be measured with a resolution of about $50 \mathrm{~m}$. The muon system is essential for momentum measurements and triggering of muons. For muons with $p_{T}>40 \mathrm{GeV}$ the momentum resolution obtained with the MS is better than the resolution obtained by the ID. For lower $p_{T}$ muons the ID measurements will determine the momentum resolution but measurements from the MS are still required to identify ID tracks as muons.

The ATLAS B-triggers used to select $B_{s}^{0} \rightarrow J / \psi \phi$ and $B_{d}^{0} \rightarrow J / \psi K^{* 0}$ decays are based on the identification of a $J / \psi \rightarrow \mu^{+} \mu^{-}$decay, with either a $4 \mathrm{GeV} p_{T}$ threshold for both muons or an asymmetric configuration that applies a higher $p_{T}$ threshold $(4-10 \mathrm{GeV})$ on one of the muons and a looser muon-identification requirement ( $p_{T}$ threshold below $4 \mathrm{GeV}$ ) on the other one.

\section{Reconstruction and candidate selection}

\subsection{Selection of $B_{s}^{0}, B_{d}^{0}$ and $J / \psi$ candidates}

Alongside good quality data coming from the fully operational muon, tracking and trigger systems, events are required to pass the following additional criteria. The event must contain at least one reconstructed primary vertex built with at least four associated ID tracks in order to be considered as a collision candidate. Tracks are required to have at least one hit in the pixel detector and at least four hits in the semiconductor tracker. The event must contain at least one pair of oppositely charged muon candidates that are reconstructed using two algorithms as described in Ref. [7]. The muons are not required to match the object(s) that fired the trigger. No cuts on the $p_{T}$ of the muons are applied other than the implicit ones in the muon reconstruction.

The muon pair tracks are refitted to a common vertex [8] and accepted for further consideration if the fit results in a $\chi^{2} /$ n.d.o.f. $<10$. The invariant mass is calculated from the refitted track parameters. To account for a varying mass resolution the $J / \psi$ candidates are divided into three subsets according to the pseudorapidity $\eta$ of the muons. A maximum likelihood fit is used to extract the $J / \psi$ mass and the corresponding resolution for these three subsets, using the method described in [9]. When both muons have $|\eta|<1.05$ the accepted signal candidates are from a region (2959-3229) MeV. When one muon has $1.05<|\eta|<2.5$ and the other muon $|\eta|<1.05$, the corresponding signal region is (2913-3273) MeV. For the third subset, where both muons have $1.05<|\eta|<2.5$, the signal region is (2852-3332) MeV. In each case, the signal region is defined to retain $99.8 \%$ of the $J / \psi$ signal identified in the fits. 


\subsection{Selection specific to $B_{d}^{0} \rightarrow J / \psi K^{* 0}$}

The $K^{* 0}$ candidates are reconstructed by selecting all pairs of oppositely charged tracks that have not been previously identified as muons, with $p_{T}>0.5 \mathrm{GeV}$ and $|\eta|<2.5$. Together with the two tracks from the $J / \psi \rightarrow \mu^{+} \mu^{-}$decay, these are fitted to a common vertex. In the fit the two muon tracks are constrained to the PDG $J / \psi$ mass value of $(3096.916 \pm 0.011) \mathrm{MeV}$ [10]. These quadruplets of tracks are accepted for further selections if the fit has a $\chi^{2} /$ n.d.o.f. $<2.5$. The new track parameters resulting from the vertex fit are used to calculate the invariant mass of $K^{* 0}$ candidates under the assumption that they are $K^{+} \pi^{-}$hadrons. These pairs are assumed to be from $K^{* 0}$ decays if the invariant mass falls within the interval (846946) $\mathrm{MeV}$ and the transverse momentum of the $K^{* 0}$ candidate is greater than $2.5 \mathrm{GeV}$. No attempt to use particle identification for the kaon or pion is made in the current study. For each candidate, both $K^{ \pm} \pi^{\mp}$ combinations are tested and in cases when both combinations satisfy the mass criterion, the one closest to the $K^{* 0}$ is chosen. In cases where more than one candidate per event is found, only the $B_{d}^{0}$ candidate with the lowest $\chi^{2} /$ n.d.o.f. is retained. The $B_{d}^{0}$ candidates with invariant mass from the interval (5159-5399) $\mathrm{MeV}$ are selected for the further analysis.

\subsection{Selection specific to $B_{s}^{0} \rightarrow J / \psi \phi$}

The $\phi \rightarrow K^{+} K^{-}$candidates are reconstructed from all pairs of oppositely charged tracks with $p_{T}>0.5 \mathrm{GeV}$ and $|\eta|<2.5$, which are not identified as muons. $B_{s}^{0} \rightarrow J / \psi\left(\mu^{+} \mu^{-}\right) \phi\left(K^{+} K^{-}\right)$ candidates are selected by fitting the tracks from each combination of $J / \psi \rightarrow \mu^{+} \mu^{-}$and $\phi \rightarrow K^{+} K^{-}$to a common vertex. The two muon tracks are constrained to the PDG $J / \psi$ mass. These quadruplets of tracks are assumed to be from $B_{s}^{0} \rightarrow J / \psi\left(\mu^{+} \mu^{-}\right) \phi\left(K^{+} K^{-}\right)$decays if the fit results in a $\chi^{2} /$ n.d.o.f. $<2$, the fitted $p_{T}$ of each track from $\phi \rightarrow K^{+} K^{-}$is greater than $1 \mathrm{GeV}$ and the invariant mass of the track pairs (under the assumption that they are kaons) falls within the interval $1009 \mathrm{MeV}<m\left(K^{+} K^{-}\right)<1031 \mathrm{MeV}$. If there is more than one $B_{s}^{0}$ candidate per event then the candidate with the lowest $\chi^{2} / n . d . o . f$. is chosen.

\section{Method of fit}

An unbinned maximum-likelihood fit is performed to extract the $B_{s}^{0}\left(B_{d}^{0}\right)$ meson mass and lifetime. The likelihood function is defined by:

$$
L=\prod_{i=1}^{N}\left(f_{\text {sig }} \mathcal{M}_{\text {sig }}\left(m_{i}\right) \mathcal{T}_{\text {sig }}\left(t_{i}\right)+\left(1-f_{\text {sig }}\right) \mathcal{M}_{b k g}\left(m_{i}\right) \mathcal{T}_{b k g}\left(t_{i}\right)\right)
$$

where $f_{\text {sig }}$ is the fraction of the signal events of the total number of events, $N$. $\mathscr{M}_{\text {sig }}$ and $\mathscr{M}_{\text {bkg }}$ are probability density functions (PDF) that model signal and background mass distributions. The terms $\mathcal{T}_{s i g}$ and $\mathcal{T}_{b k g}$ describe the decay time distributions of the signal $B_{s}^{0} \rightarrow J / \psi \phi$ $\left(B_{d}^{0} \rightarrow J / \psi K^{* 0}\right.$ ) and backgrounds $b \bar{b} \rightarrow J / \psi X$ and $p p \rightarrow J / \psi X$, respectively. The input variables to the maximum likelihood fit extracted from data are the proper decay time $t_{i}$, its 
uncertainty $\delta_{t i}$, the mass $m_{i}$ and its uncertainty $\delta_{m i}$ for each $B_{s}^{0}\left(B_{d}^{0}\right)$ candidate passing the selections.

For the signal, the mass is modelled with a Gaussian distribution and the proper decay time distribution of the $B$ candidates is modelled as an exponential function convolved with the proper decay time resolution function.

For the background, the mass distribution is modelled with a linear function, while the proper decay time is modelled depending of the source expected to contribute to the background. For the background containing the promptly produced $J / \psi$, the proper decay time is described by the resolution function; for the background candidates from non-prompt $\mathrm{J} / \mathrm{\psi}$ production the proper decay time is described by the sum of the exponential functions, each convolved with the resolution function. Subsequently, all components of the time background PDFs are summed up, relative to their contributions.

The following parameters are left free in the fit: the fraction of the signal events in the mass region of the fit $\left(f_{\text {sig }}\right)$, the fitted $B$ meson mass $\left(m_{B}\right)$ and lifetime $\left(t_{B}\right)$, the slope of the linear background, two scale factors accounting for a difference between measured per-candidate $m_{i}$ $\left(t_{i}\right)$ errors and the overall mass (proper decay time) resolution and the lifetimes characterising background components of the exponential shape.

The values of the free parameters and a covariance matrix returned by the fit are used to calculate the number of $B$ meson signal decays $N_{\text {sig }}$, the mass resolution $\sigma_{m}$ and the number of background events $N_{b k g}$ in the mass interval $m_{B} \pm 3 \sigma_{m}$, where the mass resolution $\sigma_{m}$ is defined by FWHM of the $B$ mass distribution. The uncertainty on $\sigma_{m}$ is calculated using the covariance matrix of the fit. The number of background events $N_{b k g}$ in the mass interval $m_{B} \pm 3 \sigma_{m}$ and its error are calculated from the fraction of the signal events in the mass region of the fit, the slope of the linear background and the total number of events.

As the available statistics grows it will be possible to add additional terms to the maximum likelihood fit to include decay angles in order to perform an angular analysis, thus allowing extraction of the physics parameters of interest.

\section{Results}

\subsection{Fit results}

The likelihood function defined in Section 4. is used in the simultaneous mass and lifetime fit performed on the $B$ meson candidates selected by criteria described in Section 3. Figure 1 shows the invariant mass and proper decay time distributions for the selected $B_{d}^{0}$ candidates, overlaid with the fit functions for mass and lifetime obtained by the fit method described in Section 4. Figure 2 shows the invariant mass and proper decay time distributions for the selected $B_{s}^{0}$ candidates. The results of the simultaneous mass and lifetime fit is projected onto the distributions. The results of the key parameters of the mass and lifetime fits for $B_{d}^{0}$ and $B_{s}^{0}$ mesons are summarised in Table 1. The errors given in Table 1 are statistical only. Systematic effects important for this analysis are summarised and evaluated in the next section. 
Table 1: Fit parameters determined from the simultaneous mass and lifetime fits of $B_{d}^{0}$ and $B_{s}^{0}$ candidates. Errors are statistical [11].

\begin{tabular}{ccccc}
\hline & $t_{B}[\mathrm{ps}]$ & $m_{B}[\mathrm{MeV}]$ & $\sigma_{m}[\mathrm{MeV}]$ & $N_{\text {sig }}$ \\
\hline$B_{d}^{0}$ & $1.51 \pm 0.04$ & $5279.0 \pm 0.8$ & $34.3 \pm 0.9$ & $2750 \pm 90$ \\
$B_{s}^{0}$ & $1.41 \pm 0.08$ & $5363.7 \pm 1.2$ & $24.8 \pm 1.2$ & $463 \pm 26$ \\
\hline
\end{tabular}

\subsection{Systematic uncertainties}

The goal of this analysis is to validate the lifetime measurement technique in preparation for $\mathrm{CP}$ violation studies and those systematic effects that are believed to be the most important are considered: modelling of signal and background in likelihood fit, fitting procedure, size of the mass window, time uncertainty model, choice of primary vertex and alignment of the Inner Detector. For the data used in this analysis, no systematic uncertainty is assigned due to the fitting procedure and the primary vertex selection method. A summary of all assigned systematic uncertainties is given in Table 2 .

Table 2: Systematic uncertainties in the $B_{d}^{0}$ and $B_{s}^{0}$ lifetime measurement [11].

\begin{tabular}{lcc}
\hline Source of systematics & \multicolumn{2}{c}{ Systematic uncertainty } \\
& $\delta_{\text {syst }}\left(t_{B d}\right)[\mathrm{ps}]$ & $\delta_{\text {syst }}\left(t_{B s}\right)[\mathrm{ps}]$ \\
\hline Modelling signal, background & 0.01 & 0.01 \\
Time uncertainty model & 0.03 & 0.03 \\
Mass window & 0.01 & 0.02 \\
Alignment & 0.03 & 0.03 \\
\hline Total, quadratic sum & 0.04 & 0.05 \\
\hline
\end{tabular}

\subsection{Discussion of results}

The results demonstrate that a simultaneous unbinned maximum likelihood fit to mass and lifetime using per-candidate errors can be used to separate $B_{s}^{0}$ and $B_{d}^{0}$ signals from background. A single exponential fit can be applied to the signal events. The estimated sizes of the systematic errors on the $B_{s}^{0}$ and $B_{d}^{0}$ lifetimes are comparable to their statistical errors.

Measured masses and lifetimes of $B$ mesons agree with the world averages within the precision of the current analysis. Since the $B_{s}^{0} \rightarrow J / \psi \phi$ final state is dominated by the shorterlived component of $B_{s}^{0}$ mesons, the single exponential model applied in the current study is inherently biased. However, within the current precision the fitted value agrees well with the world average $1 / \Gamma_{s}=1 /\left(\Gamma_{L}-\Gamma_{H}\right) / 2$ [10]. The lifetime value returned by the fit is closer to the shorter lifetime, $1 / \Gamma_{L}=1.408_{-0.030}^{+0.033}$ ps [12]. 

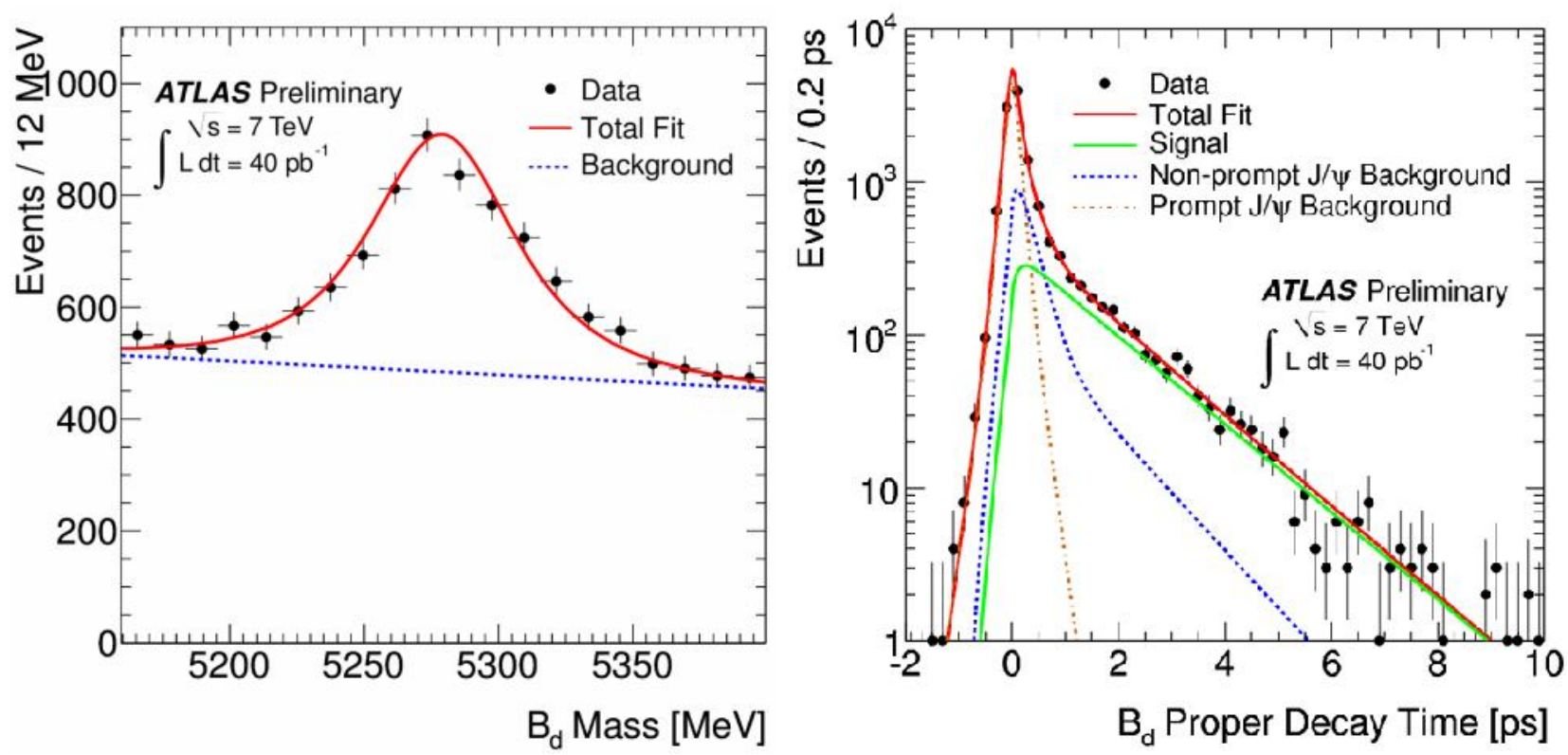

Figure 1: Invariant mass (left) and proper decay time (right) distributions of reconstructed $B_{d}^{0} \rightarrow J / \psi K^{* 0}$ decay candidates [11].
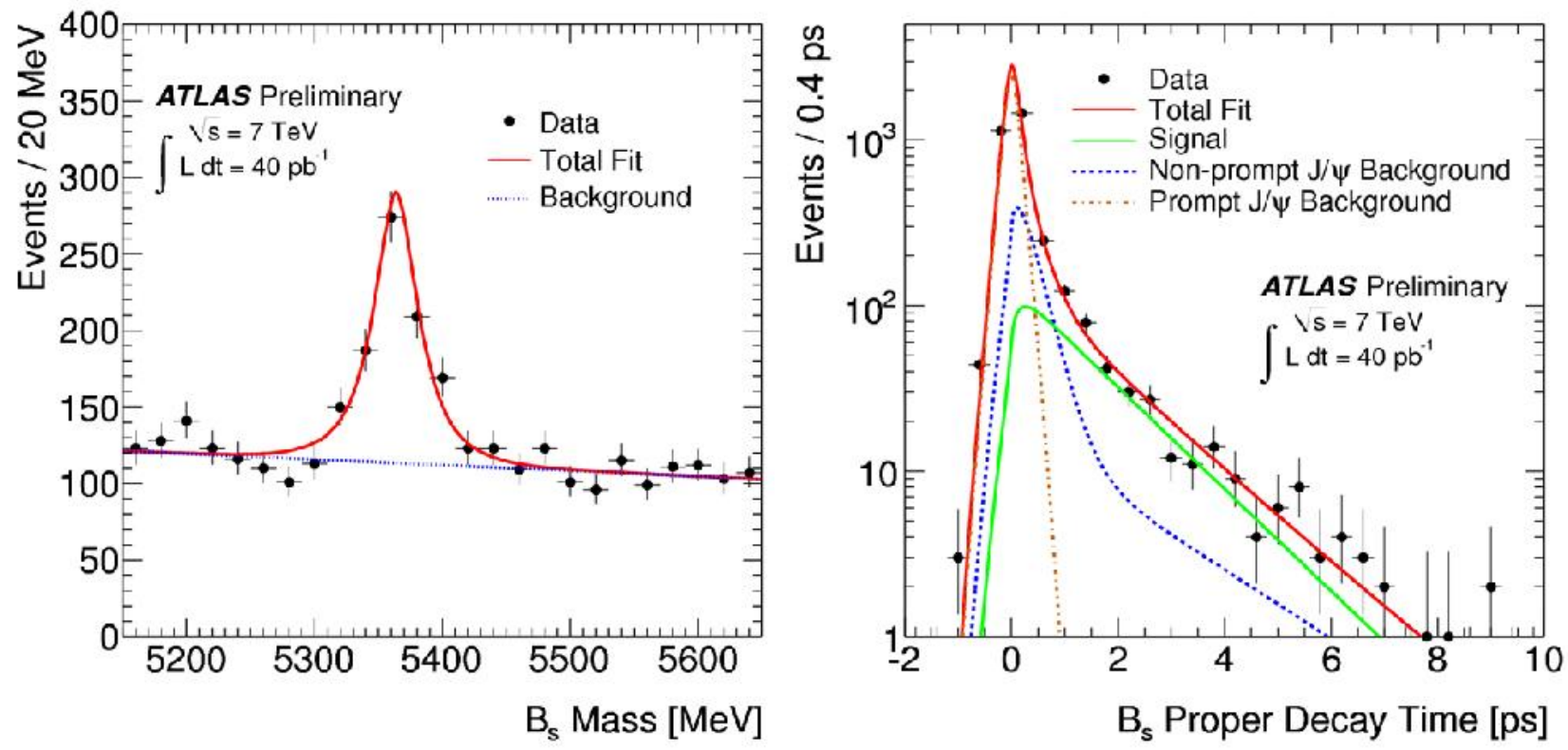

Figure 2: Invariant mass (left) and proper decay time (right) distributions of reconstructed $B_{s}^{0} \rightarrow J / \psi \phi$ decay candidates [11]. 


\section{Conclusion}

The performance of the ATLAS detector towards CP violation measurements has been tested. Using $40 \mathrm{pb}^{-1}$ of $p p$ collision data at $7 \mathrm{TeV}$ the $B_{s}^{0}$ and $B_{d}^{0}$ mesons are reconstructed from their decays $B_{s}^{0} \rightarrow J / \psi \phi$ and $B_{d}^{0} \rightarrow J / \psi K^{* 0}$. The masses and proper decay times of $B_{s}^{0}$ and $B_{d}^{0}$ candidates are measured using a simultaneous unbinned maximum likelihood fit. The results show agreement with the world average values confirming the validity of the methods for reconstructing primary and secondary vertices and measuring the transverse decay length.

This analysis validate the capability of the ATLAS experiment to measure masses and lifetimes of both $B_{s}^{0}$ and $B_{d}^{0}$ mesons with high precision and thus to meet the requirements necessary to perform precision $\mathrm{CP}$ violation measurements.

\section{References}

[1] A. Lenz and U. Nierste, Theoretical update of $B_{s}^{0}-\overline{B_{s}^{0}}$ mixing, JHEP 06 (2007) 072, arXiv:hep-ph/0612167.

[2] A.S. Dighe, I. Dunietz, H.J. Lipkin and J.L. Rosner, Angular Distributions and Lifetime Differences in $B_{s}^{0} \rightarrow J / \psi \phi$ decays, Phys. Lett. B369 (1996) 144.

[3] A.S. Dighe, I. Dunietz and R. Fleischer, Extracting CKM Phases and $B_{S}^{0}-\overline{B_{S}^{0}}$ Mixing Parameters from Angular Distributions of Non-Leptonic B Decays, Eur. Phys. J. C6 (1999) 647.

[4] I. Dunietz, $B_{s}^{0}-\overline{B_{s}^{0}}$ Mixing, CP violation, and extraction of CKM phases from untagged $B_{s}^{0}$ data samples, Phys. Rev. D52 (1995) 3048.

[5] ATLAS Collaboration, The ATLAS Experiment at the CERN Large Hadron Collider, JINST 3 (2008) S08003.

[6] ATLAS Collaboration, Performance of primary vertex reconstruction in proton-proton collisions at $\sqrt{s}=7 \mathrm{TeV}$ in the ATLAS experiment, ATLAS-CONF-2010-069, http://cdsweb.cern.ch/record/1281344.

[7] ATLAS Collaboration, Observation of the $B_{d}^{0}$ and $B_{s}^{0}$ mesons in the decays $B_{d}^{0} \rightarrow J / \psi K^{* 0}$ and $B_{s}^{O} \rightarrow J / \psi \phi$ in ATALS, ATLAS-CONF-2011-050, http://cdsweb.cern.ch/record/1341815.

[8] ATLAS Collaboration, VKalVrt - package for vertex reconstruction in ATLAS, ATL-PHYS-2003-031, http://cdsweb.cern.ch/record/685551.

[9] ATLAS Collaboration, First observation of the $\mathrm{J} / \psi \rightarrow \mu^{+} \mu^{-}$resonance in ATLAS pp collisions at $\sqrt{s}=7 \mathrm{TeV}$, ATLAS-CONF-2010-045, http://cdsweb.cern.ch/record/1277685.

[10] K. Nakamura et al., (Particle Data Group), J. Phys. G 37 (2010) 075021.

[11] ATLAS Collaboration, Measurement of the $B_{d}^{0}$ and $B_{s}^{0}$ lifetimes in the decay modes $B_{d}^{0} \rightarrow J / \psi K^{* 0}$ and $B_{s}^{O} \rightarrow J / \psi \phi$ in ATLAS, ATLAS-CONF-2011-092, http://cdsweb.cern.ch/record/1363779.

[12] O. Schneider et al., $B_{s}^{0}-\overline{B_{s}^{0}}$ Mixing, J. Phys. G 37 (2010) 075021. 